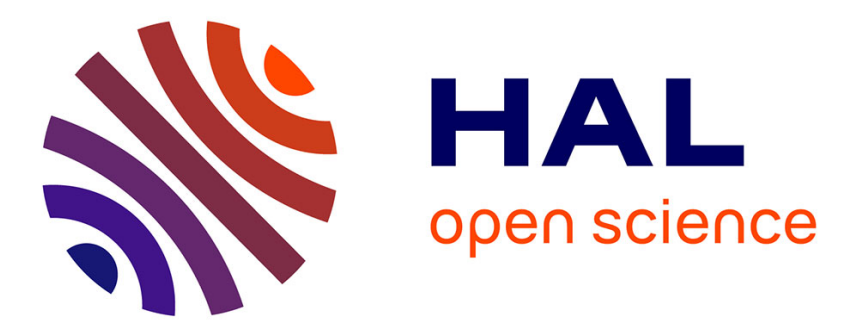

\title{
Structural Basis for $\alpha$-Helix Mimicry and Inhibition of Protein-Protein Interactions with Oligourea Foldamers
}

Léonie Cussol, Laura Mauran-ambrosino, Jérémie Buratto, Anna Belorusova, Maxime Neuville, Judit Osz, Sébastien Fribourg, Juliette Fremaux, Christel Dolain, Sébastien Goudreau, et al.

\section{To cite this version:}

Léonie Cussol, Laura Mauran-ambrosino, Jérémie Buratto, Anna Belorusova, Maxime Neuville, et al.. Structural Basis for $\alpha$-Helix Mimicry and Inhibition of Protein-Protein Interactions with Oligourea Foldamers. Angewandte Chemie International Edition, 2020, 10.1002/anie.202008992 . hal-03039251

\section{HAL Id: hal-03039251 \\ https://hal.science/hal-03039251}

Submitted on 8 Dec 2020

HAL is a multi-disciplinary open access archive for the deposit and dissemination of scientific research documents, whether they are published or not. The documents may come from teaching and research institutions in France or abroad, or from public or private research centers.
L'archive ouverte pluridisciplinaire HAL, est destinée au dépôt et à la diffusion de documents scientifiques de niveau recherche, publiés ou non, émanant des établissements d'enseignement et de recherche français ou étrangers, des laboratoires publics ou privés. 


\title{
Structural basis for $\alpha$-helix mimicry and inhibition of protein-protein interactions with oligourea foldamers
}

\author{
Léonie Cussol,a,+ Laura Mauran,a,b,+ Jérémie Buratto,a Anna Y Belorusova,c Maxime Neuville,a,b \\ Judit Osz,c Sébastien Fribourg,d Juliette Fremaux,b Christel Dolain, a Sébastien R. Goudreau,b \\ Natacha Rochel,c Gilles Guicharda*
}

[a] Dr. L. Cussol, Dr. L. Mauran, Dr. J. Buratto, M. Neuville, Dr. C. Dolain, Dr G. Guichard Univ. Bordeaux, CNRS, Bordeaux INP, CBMN, UMR 5248

Institut Européen de Chimie et Biologie,

2 rue Robert Escarpit, F-33607 Pessac, France

E-mail: g.guichard@iecb.u-bordeaux.fr

[b] Dr. L. Mauran, Dr. J. Fremaux, Dr. S. R. Goudreau

UREKA Pharma SAS,

2 rue Robert Escarpit, F-33607 Pessac, France

[c] Dr. A.Y. Belorusova, Dr. J. Osz, Dr. N. Rochel

Institut de Génétique et de Biologie Moléculaire et Cellulaire (IGBMC), INSERM, U1258/CNRS, UMR

7104/Univ. Strasbourg, 67404 Illkirch, France.

[d] Dr S. Fribourg

ARNA Laboratory, INSERM U1212, UMR CNRS 5320, Univ. Bordeaux, Bordeaux, France

[+] These authors contributed equally to this work.

Supporting information for this article is given via a link at the end of the document.

\begin{abstract}
Efficient optimization of a peptide lead into a drug candidate frequently needs further transformation to augment properties such as bioavailability. Among the different options, foldamers, sequence-based oligomers with precise folded conformation, have emerged as a promising technology. Here, we introduce oligourea foldamers to reduce the peptide character of inhibitors of protein-protein interactions (PPI). However, the precise design of such mimics is currently limited by the lack of structural information on how these foldamers adapt to protein surfaces. We now report a collection of X-ray structures of peptide-oligourea hybrids in complex with ubiquitin ligase MDM2 and vitamin D receptor and show how such hybrid oligomers can be designed to bind with high affinity to protein targets. This work should enable the generation of more effective foldamer-based disruptors of PPIs in the context of peptide lead optimization.
\end{abstract}




\section{Introduction}

Peptides have re-emerged as key molecules in modern drug discovery (new 'modalities'[1]) owing to some specific advantage such as their high molecular and structural diversity, effective synthesis methods, and ease of generating sequences with high affinity and selectivity for difficult biological targets.[2] However, there is commonly a need for further optimizing standard peptides consisting of proteinogenic amino acids as they generally possess poorly defined conformation, short in vivo halflife and poor membrane permeability.[2c, 3] This incited chemists to develop innovative approaches to address these challenges, among which constrained peptides have gained momentum with several drug candidates at different stages of clinical trials.[4] In particular, considerable effort has been devoted to the stabilization and mimicry of helical secondary and tertiary structure motifs as they are frequently involved at the interface of protein-protein interactions (PPIs), a prominent source of biological targets.[5] Multiple strategies were developed for that purpose, including amino acid replacement using non canonical residues, macrocyclization, and backbone modifications.[6] The foldamer strategy which consists in designing sequence-specific synthetic oligomers that fold into well-defined structures[7] is appealing as such backbone modifications might increase the resistance of the peptide, and improve its physicochemical characteristics while preserving affinity for the target.

Yet, examples of bioactive peptides modified using foldamer technologies remain scarce due to both (i) the difficulty to identify effective mimics that can retain essential features of peptide secondary structures including correct orientation of key side chains, and (ii) the need for high resolution structural information to guide structure-activity based studies. In few cases, well-designed foldamers led to significant improvements of the pharmaceutical properties of the cognate peptides. $\alpha / \beta$-Peptides developed by Gellman as modulators of PPIs or receptor ligands are highly significant in that respect.[7d, 7f-h] Recently, we showed the first example of peptide-oligourea hybrids as analogues of class B GPCR peptide ligands with improved activity in vivo.[8] N, $\mathrm{N}^{\prime}$-linked oligoureas have distinctive properties: 1 ) they are sequence-defined and readily accessible; 2 ) they can form robust helical structures akin to the $\alpha$-helix; [9]; 3) they show high resistance to proteolysis;[10] and 4) they can be interfaced with peptides to generate regular hybrid helices;[11] However, the main challenge when replacing a $\alpha$-helical peptide by its ureido counterpart is to take into account the structural differences between the two helices, i.e. the smaller number of residues per turn, the smaller rise per turn and the larger diameter of the oligourea helix (Figure 1). In this context, the lack of high resolution structural information on oligoureas or peptide-oligoureas hybrids bound to a protein target represents a serious limitation to their utilization. The purpose of this study was to gain structural insights into the interaction of oligoureas with protein surfaces and delineate some principles that in turn could be used to accelerate the discovery of peptide/oligourea hybrids as modulators of PPIs.

Two representative PPIs involving short peptide helices were selected: 1) the p53-MDM2 interaction and 2) the SRC-vitamin D receptor (VDR) interaction. 
A

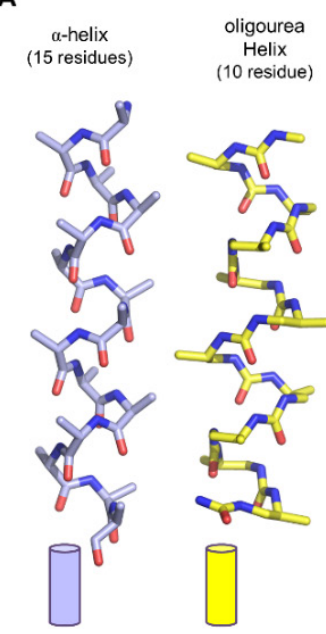

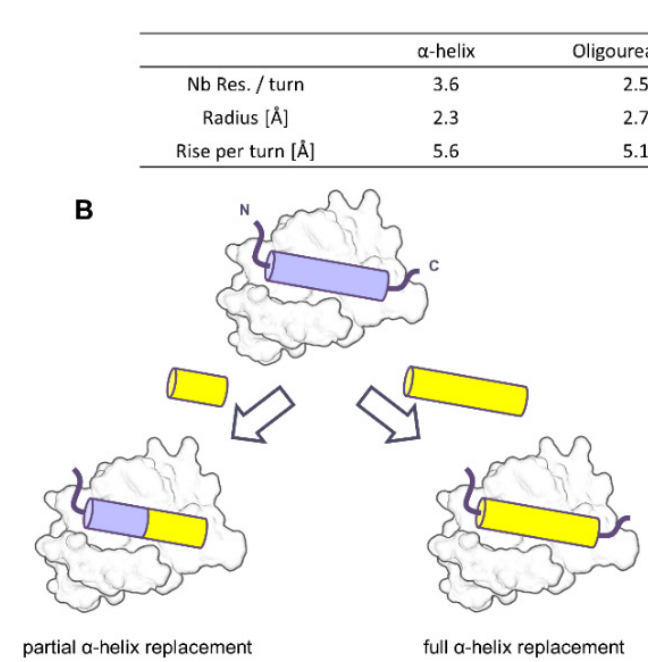

C

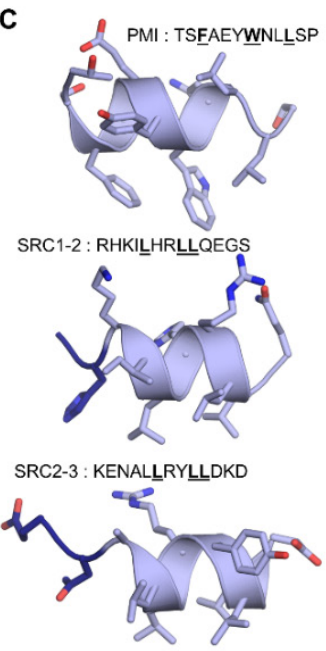

Figure 1. General principle for $\alpha$-helix mimicry with oligourea foldamers. A) Comparison of the peptide $\alpha$-helix ( $C$ atoms in light blue) and oligourea 2.5 helix ( $C$ atoms in yellow). B) Schematic representation showing how oligourea helices can be used to replace $\alpha$-helical portions in target peptides to generate oligourea-peptide hybrids as PPI inhibitors. Ureido residues are denoted Xu and Xu $\alpha$ by analogy to the one letter code of $\alpha$-amino acids. C) Peptides PMI, SRC1-2, and SRC2-3 used as starting points to design corresponding peptide/oligourea ligands. Bound conformation to their respective targets, namely MDM2 (PDB ID : 3EQS[12]) and VDR (PDB ID : 2HC4[13] and 5H1E[14]).

Both $\mathrm{p} 53$ and VDR are transcription factors. The tumor suppressor p53 is a protein that regulates apoptosis in response to various stresses and which is negatively regulated by the ubiquitin ligase MDM2. Inhibiting or degrading MDM2 to restore wild-type p53 activity in tumors that overexpress MDM2 is a potential strategy for cancer treatment.[15] The VDR is a member of the nuclear hormone receptor (NHR) family which is implicated in the regulation of many biological functions including bone homeostasis, cell growth, and immunity.[16] Transcriptional activity of VDR upon binding to 1,25-dihydroxyvitamine D3 (1,25-(OH)2D3 or calcitriol) its natural ligand requires the molecular association of the ligand binding domain (LBD) with coactivators including steroid receptor coactivator (SRC) family members (SRC1, SRC2, SRC3). Inhibiting NHR-SRC interaction is a potential strategy to treat cancer associated with elevated expression of SRC.[17]

Herein, we show that peptides with oligourea inserts may be designed to retain high affinity for their protein target and report six crystal structures of peptide/oligourea hybrids bound to their respective protein target. These high resolution structures are particularly revealing in showing that all hybrids adopt a regular helical conformation upon binding to the target protein and in suggesting how the protein may accommodate non canonical helical backbones.

\section{Results and Discussion}

\section{General considerations on the design of peptide-oligourea hybrids}

The selected targets MDM2 and VDR differ by their mode of helix recognition and by the length of the bound $\alpha$-helix ( 7 and 9 residues for SRC- and p53-derived peptides) thus allowing different peptide ? oligourea replacement strategies to be explored (Figure 1B). Previously, we scanned the GLP-1 sequence with triureas inserts as tetrapeptide mimics to identify replacements that would retain agonist activity while potentiating pharmacokinetics properties.[8] In this work, we thought to apply this strategy of partial replacement by incremental extension of the oligourea segment starting from the C-terminus to create p53 mimics with increased resistance to proteolytic degradation. In the case of VDR, the interaction of the LXXLL motif of coactivators with the LBD is mediated by both a 
hydrophobic cleft which accommodates the Leu side chains and a charge clamp formed by conserved charged residues in the LBD (e.g. E446 and K274 in zebrafish VDR (zVDR)) that contact terminal main chain amides of the helix. Here the challenge is thus to replace the central $\alpha$-helix by an oligourea helix of precise length that would retain this general binding mode including electrostatic interactions with the charge clamp.

\section{Short oligourea-peptide hybrids as potent disruptors of p53-MDM2 interaction}

Starting from PMI, a dodecapeptide selected from phage display libraries, with affinity for MDM2 in the $\mathrm{nM}$ range (Figure $1 \mathrm{C}),[12,18]$ we set out to design analogues by gradually introducing ureido units to replace $\mathrm{C}$-terminal region including $\mathrm{L} 10$ and $\mathrm{W} 7$ which make key contribution to MDM2 binding, and to create a capping box at the C-terminus of the helix.[12] The target oligomers were prepared by solid-phase synthesis (SPS) using Fmoc chemistry for the peptide fragments and azidoalkyl succinimidyl carbamate monomers for the incorporation of ureido residues.[8, 19]

Table 1. Sequences of oligourea-peptide hybrids 1-9 derived from PMI and binding to MDM2 as determined by TR-FRET assay

\begin{tabular}{|c|c|c|c|c|c|c|c|c|c|c|c|c|c|c|}
\hline \multirow[t]{2}{*}{ Compound } & \multicolumn{12}{|c|}{ Sequence ${ }^{[a]}$} & \multirow[t]{2}{*}{$I_{5} \mathrm{C}^{[\mathrm{b}]}(\mathrm{nM})$} & \multirow[t]{2}{*}{$S E^{[c]}(n M)$} \\
\hline & 1 & 2 & 3 & 4 & 5 & 6 & 7 & 8 & 9 & 10 & 11 & 12 & & \\
\hline Nutlin 3a & & & & & & & & & & & & & $21^{[\mathrm{d}]}$ & $2.4^{[\mathrm{e}]}$ \\
\hline PMI & $\mathrm{T}$ & S & $\mathrm{F}$ & A & $\mathrm{E}$ & $Y$ & W & $\mathrm{N}$ & L & L & $S$ & $P$ & 9.5 & 0.5 \\
\hline $\mathrm{PMI}(1-10)$ & $\mathrm{T}$ & $S$ & $\mathrm{~F}$ & $A$ & $E$ & $\mathrm{Y}$ & w & $\mathrm{N}$ & L & L & - & - & 14 & 1.6 \\
\hline 1 & $\mathrm{~T}$ & s & $\mathrm{F}$ & $A$ & $E$ & $Y$ & w & $A^{u}$ & $A^{u}$ & $L^{u}$ & - & - & $39^{[f]}$ & $2.2^{[\mathrm{e}]}$ \\
\hline 2 & $\mathrm{~T}$ & $S$ & $\mathrm{~F}$ & A & $E$ & $Y$ & w & $A^{u}$ & $A^{u}$ & $A^{u}$ & - & - & 232 & 19 \\
\hline 3 & $\mathrm{~T}$ & $S$ & $\mathrm{~F}$ & A & $\mathrm{E}$ & Y & w & $A^{u}$ & $A^{u}$ & $\mathrm{Nle}^{\mathrm{u}}$ & - & - & 67 & 4.6 \\
\hline 4 & $\mathrm{~T}$ & $\mathrm{~s}$ & $\mathrm{~F}$ & A & $E$ & $Y$ & w & $A^{u}$ & $A^{u}$ & $\mathrm{~V}^{\mathrm{u}}$ & - & - & $24^{[\mathrm{ff}]}$ & $1.8^{[\mathrm{e}]}$ \\
\hline 5 & $\mathrm{~T}$ & $\mathrm{~s}$ & $\mathrm{~F}$ & A & $E$ & $\mathrm{Y}$ & $\mathbf{W}^{u}$ & $A^{u}$ & Vua & $A^{u}$ & - & - & 529 & 42 \\
\hline 6 & $\mathrm{~T}$ & $S$ & $\mathrm{~F}$ & A & $E$ & $\mathrm{Y}$ & $\mathbf{W}^{u}$ & $A^{u}$ & Lua & $A^{u}$ & - & - & 60 & 10 \\
\hline 7 & $\mathrm{~T}$ & $S$ & $\mathrm{~F}$ & A & $E$ & Y & $\mathbf{W}^{u}$ & $A^{u}$ & $\mathrm{Lu}^{\mathrm{u}}$ & $A^{u}$ & - & - & 1443 & 141 \\
\hline 8 & $\mathrm{~T}$ & $\mathrm{~s}$ & $\mathrm{~F}$ & A & $E$ & Y & $\mathbf{W}^{u}$ & $A^{u}$ & $A^{\text {ua }}$ & $A^{u}$ & - & - & 1806 & 308 \\
\hline 9 & $\mathrm{~T}$ & $\mathrm{~s}$ & $\mathrm{~F}$ & A & $E$ & $Y$ & $\mathbf{W}^{u}$ & $A^{u}$ & Lu $^{\text {u }}$ & - & - & - & 77 & 4.9 \\
\hline
\end{tabular}

Yields after purification varied between 10-30\% (Supporting Information (SI)). The interaction between $\mathrm{PMI}$ analogues and MDM2 was quantified using a time resolved fluorescence energy transfer (TR-FRET) assay[20] by measuring the suppression of the interaction between recombinant p53 and MDM2 upon addition of increasing concentrations of the peptide mimics (Table 1; Figures 
S1-S12). (-)-Nutlin 3a, PMI and PMI(1-10) were used as controls. The first hybrids were prepared by replacing the five amino acids from position 8 to 12 (NLLSP) with a simple triurea motif AuAuXu. The residue $\mathrm{Xu}$ which was intended to mimic L10 was varied from Lu to Au, Nleu and $\mathrm{Vu}$ (compounds 1-4) in order to investigate the impact of the side chain on the binding. Interestingly, the first compound in this series (1) was found to be only 4 times less potent than PMI with an IC50 of $39 \mathrm{nM}$. The importance of the Xu residue was further confirmed by the significant drop in affinity observed for hybrid 2 bearing a smaller Me side chain. Remarkably, compound $4(\mathrm{Vu}, \mathrm{IC} 50=24 \mathrm{nM})$ was the tightest binder in the series and shows a binding profile similar to (-)-nutlin-3a, indicating that an $\mathrm{Pr}$ side chain is a better mimic of L10 when introducing an oligourea backbone at the C-terminus of PMI.

A crystal structure of 4 in complex with MDM2 was obtained (vide infra) which allowed us to rationalize this finding, validate our models and design a new series of analogues with further replacement of the key amino acid W7. The idea was to replace $W 7$ in 4 by a Wu residue to gain additional protection towards enzymatic degradation as cleavage after W7 has been reported.[21] However, an overlay of a model hybrid with the crystal structure of bound PMI suggested that introducing a WuAuAuVu sequence would not fit well in the p53-binding cavity as the Vu side chain would be located too far from the leucine binding site and would clash with the protein surface (Figure S13). Instead, the model was supporting a design with $W u$ and $V u$ in a $(i, i+2)$ relationship. Closer inspection of the model also suggested that the regular substitution pattern (on the $B C$ ) in $\mathrm{Vu}$ was not optimal and that moving the iPr moiety to the second carbon $(\alpha \mathrm{C})$ of the ureido residue would provide a better mimic. We have shown previously that combining a shift of the substitution position ( $\mathrm{C}$ ( $\mathrm{a} \alpha$ ) with an inversion of the configuration retains the helical conformation of the oligomer and allows fine tuning of the side-chain distribution at the surface of the helix.[9] The resulting hybrid 5 incorporating this modification was found to retain a submicromolar affinity for MDM2 (529 nM) despite the replacement of two key side chains of PMI. Remarkably, further modification of the side chain from $\mathrm{iPr}(\mathrm{Vu \alpha}, 5)$ to $\mathrm{iBu}(\mathrm{Lu \alpha}, 6)$ gave a tighter binder with an IC50 of $60 \mathrm{nM}$. The corresponding analogue with the iBu side chain shifted to the $\beta$-carbon gave a much lower affinity $(7,1443 \mathrm{nM})$, thus supporting our binding mode hypothesis. Modifying the iBu side chain in 6 to a smaller Me group (8) led to a drop of affinity of about 30-fold confirming the critical contribution of the Lua side chain to the binding. Interestingly the last Au ureido residue is not essential and can be removed without much loss in binding affinity (see 9) allowing significant downsizing. Overall, the finding that the last 6 residues of PMI can be replaced by a short oligourea triad (9) with less than 8-fold loss of binding affinity highlights the effectiveness of oligoureas as $\alpha$ helix mimics. 
A

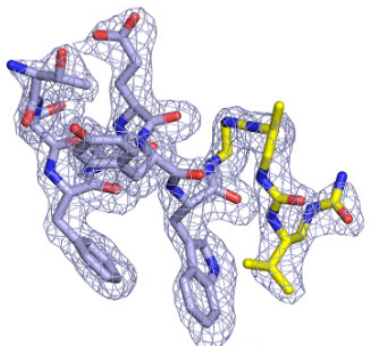

$\mathrm{D}$

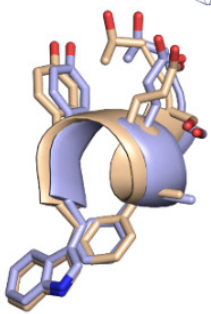

$\mathrm{E}$
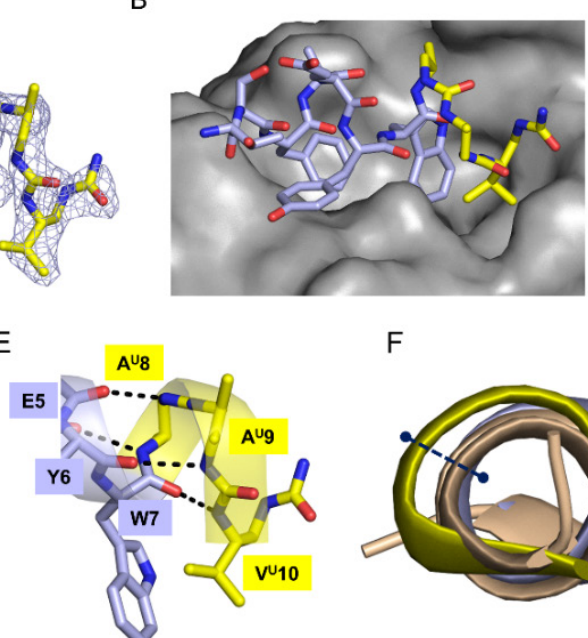

$\mathrm{F}$
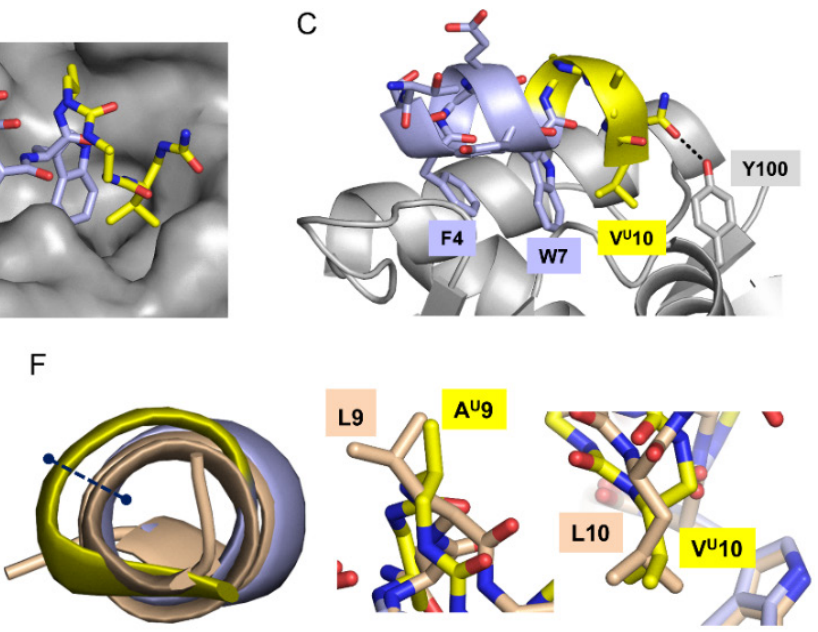

Figure 2. Crystal structure of hybrid 4 bound to MDM2. A) Electron density map of 4 contoured at $1 \sigma$ level. B) Hybrid 4 on the molecular surface of MDM2. C) Details of the H-bond between the urea carbonyl of Vu10 and the hydroxyl group of Y100. D) Overlay of the peptide parts of 4 (light blue) and PMI (wheat) in the bound conformation. E) The H-bond network at the junction between the peptide and oligourea backbones. F) (left) Overlay along the helix axis of bound PMI and hybrid 4 (ribbon). As a result of its increased diameter the oligourea helix extends by $1.8 \AA$. (center and right) Correspondence between oligourea and peptide side chains at positions 9 and 10 .

\section{Co-crystal structure of 4 bound to MDM2 and comparison with cognate peptide ligand}

To gain further insight into $\alpha$-helix mimicry with oligoureas and MDM2 recognition by oligourea/peptide hybrids reported, we determined the co-crystal structure of 4 bound to human MDM2 (residues 17-111) at 1.79 Å resolution (see Table S1 for data collection and refinement statistics). The crystal structure was found to contain two similar copies of the complex in the asymmetric unit (ASU) (Figure S14) which only differ by the orientation of few side chains in MDM2 (the root mean square deviation (r.m.s.d.) on $84 \alpha \mathrm{C}$ is $0.587 \AA$ ). The electron density map of 4 contoured at $1 \sigma$ level was well-defined and allowed for unambiguous positioning of all side chains (Figure 2A). The structure shows that bound 4 adopts a helical conformation spanning both the peptide and oligourea segments from Phe3 to Vu10 and an overall binding mode to MDM2 similar to $\mathrm{PMI}$ (Figure 2B and 2C) with almost no difference in the structure of MDM2 (Figure S15). The bound peptide chain in 4 ( $T 1$ to $W 7$ ) is largely identical to the corresponding part in bound PMI with projection of key hydrophobic side chains F3 and W7 unchanged (Figure 2D). Additional features such as the capping box at the $\mathrm{N}$-terminus of the sequence and specific Van der Waals and cation- $\pi$ interactions between $\mathrm{Y} 6$ and the surface of MDM2 are well preserved. The $\alpha$-helix is prolonged at its C-terminus by the 2.5 oligourea helix and the two helices are interconnected by an intramolecular $\mathrm{H}$ bonding network involving amide carbonyls of residues E5-W7 and urea NHs of residues Au8-Vu10 (Figure 2E).

The difference in diameter between the oligourea and peptide helices at the C-terminus of bound PMI and 4 is striking (Figure 2F). The enlarged diameter (about $1.8 \AA$ ) of the oligourea helix in 4 compared to the $\alpha$-helix in PMI accounts for a significant shift of the side chains. For example, the Me side chain of Au9 overlaps with the $\delta$ Me of L9 of PMI. Remarkably, the difference in helix geometry also explains why the iPr side chain of $\mathrm{Vu} 10$ is the best topological mimic of $\mathrm{L} 10$ as indicated by the buried surface percentage (BS\%) of the two side chains ( $100 \%$ and $99 \% \AA 2$, respectively). Additional contribution to the binding provided by the oligourea helix also comes from the $\mathrm{H}$-bond between the 
terminal urea carbonyl and $\mathrm{Y} 100$ which parallels the interaction between $\mathrm{C}=\mathrm{O}$ of $\mathrm{L} 10$ with $\mathrm{Y} 100$ in the PMI-MDM2 complex (Figure 2C). It should also be mentioned that for one of the two complexes in the ASU, the imidazole side chain of H96 in MDM2 flipped towards $\mathrm{Y} 100$ and is within a H-bond distance of the terminal urea carbonyl, suggesting a possible contribution to binding (Figure S16).

\section{Design and synthesis of coactivator-derived-peptide-oligourea hybrids and binding to VDR}

The crystal structures of liganded VDR LBD in complex with coregulatory peptides SRC1-2 (PDB ID: 2HC4,[13] 4G1Z[22]) and SRC2-3 (PDB ID: 5H1E[14]) provided a structural basis for the rational design of chimeric foldamers ( 8-11 residue long). SRC1-2 and SRC2-3 are tridecapeptides that comprise a central consensus LXXLL motif and differ by their flanking residues. Both sequences contain a hydrophobic residue (L/I) N-terminal to the LXXLL motif and bind VDR with relatively high affinity compared to other coactivators such as SRC1-1 and SRC2-1 which contain a polar residue at that position. This is explained in part by the additional hydrophobic contact at the surface of the VDR.[23] Additional polar contact may also contribute to the stronger affinity of SRC2-3 for VDR.[14]

In the first replacement strategy, we aimed at scanning the helical LXXLL motif of SRC1-2 by replacing 4 consecutive $\alpha$-residues by a triurea segment. Two analogues 10 and 11 were synthesized in which two of the four key hydrophobic residues have been replaced by ureido counterparts. In an attempt to more closely mimic the native peptide side chains and improve interaction with VDR, we synthetized 12 , an analogue of 11 with one sequence modification in the triurea segment $(\mathrm{Ku}$ ? Ru). Superimposition of the oligourea backbone on the structure of the bound coactivator peptide (Figure S17) suggests that the charge clamp of VDR LBD could also accommodate a full oligourea helix of appropriate length (i.e. 5-residues). To test this hypothesis, we designed compounds 13-18 which contain a pentaurea segment of sequence XuaLuYuVuNleu to replace the whole $\alpha$-helical portion (LLRYLLD) of SRC2-3. The linear Nleu side chain was preferred to a branched side chain at the last position to avoid possible clashes within the hydrophobic pocket. To better mimic the orientation of the first $L$ side chain which makes van der Waals contacts with the protein surface and optimize side chain spacing in the oligourea segment, we decided to introduce a Xua residue at the first position. All compounds (Table 2) were obtained using SPS (Supporting Information). We first used nano differential scanning fluorimetry to compare the thermal stability of the purified zVDR LBD upon binding to 1,25-(OH)2D3 and the hybrids (Table S2).[24] The complexes with the natural peptides containing SRC1-2 and SRC2-3 motifs exhibit a stabilization of the melting temperature $\Delta \mathrm{Tm}$ of $\sim 7^{\circ} \mathrm{C}$. The $\Delta T \mathrm{Tm}$ observed upon binding of the modified peptides to zVDR LBD vary from 1 to $6.5^{\circ} \mathrm{C}$. The smaller $\Delta \mathrm{Tm}$ was obtained with hybrid 10 in which the central helix is partially replaced (tetrapeptide ? triurea). Interestingly, three SRC2-3 analogues with a central pentaurea helix were shown to exhibit a $\triangle \mathrm{Tm}$ above $5^{\circ} \mathrm{C}$ indicating that the total replacement of the canonical LXXLL motif can be achieved with minimal consequence for the stability of the complex.

We next measured the interaction between human VDR LBD and the oligourea-peptide hybrids by microscale thermophoresis in the presence of 1,25-(OH)2D3 (Table 2 and Figure S18). Weak affinities were calculated for both hybrids 10 and 11, i.e. KD of $360 \mu \mathrm{M}$ and $215 \mu \mathrm{M}$ respectively compared to $2.3 \mu \mathrm{M}$ for the reference peptide (SRC1-2). This result suggests that either the distribution of the hydrophobic side chains is not optimal or the electrostatic interactions with the charge clamp is affected, or both. Replacement of Ku by Ru (11 12 12) led to a modest 3-fold increase in binding (KD = $64 \mu \mathrm{M})$.

A clear improvement in binding affinity was observed in the pentaurea series (13-19). Compound 13 was found to be equipotent to SRC2-3. Analogues 14 and 15 deleted from the flanking residues at 
either end of the sequence bound to VDR LBD with affinities 16- and 2.5-fold weaker than 13, respectively, supporting a substantial contribution of the $\mathrm{C}$-terminal residues to the binding. The moderate improvement in binding affinity of 16 and its fluorescently labelled (FITC) analogue (17) compared to that of 15 is consistent with a minor contribution of the side chain of the ureido residue at the $\mathrm{N}$-terminus. It is also noteworthy that extension of the urea backbone of 15 by one additional Au unit towards the N-terminus to give 9-mer 18 restores optimal binding to VDR with a KD of 1.4 $\mu \mathrm{M}$.

\section{Structural basis for $\alpha$-helix mimicry and VDR recognition by oligoureas}

With the aim to analyze the consequences of peptide ? oligourea replacements on folding and protein surface recognition, we determined the structures of the ternary complexes between zVDR LBD, 1,25-(OH)2D3 and five different hybrids, namely 10-12, 13 and 18.

Table 2. Sequences of oligourea-peptide hybrids 10-19 derived from SRC peptides and binding to hVDR LBD as determined by microscale thermophoresis

\begin{tabular}{|c|c|c|c|c|c|c|c|c|c|c|c|c|c|c|c|c|}
\hline \multirow[t]{2}{*}{ Compound } & \multicolumn{13}{|c|}{ Sequence $e^{[a]}$} & & \multirow[t]{2}{*}{$K_{D^{[b]}}(\mu \mathrm{M})$} & \multirow[t]{2}{*}{$S D^{[c]}(\mu M)$} \\
\hline & 1 & 2 & 3 & 4 & 5 & 6 & 7 & 8 & 9 & 10 & 11 & 12 & 13 & & & \\
\hline SRC1-2 & $\mathrm{R}$ & $\mathrm{H}$ & $\mathrm{K}$ & I & $\mathrm{L}$ & $\mathrm{H}$ & $\mathrm{R}$ & $\mathrm{L}$ & $\mathrm{L}$ & $Q$ & $\mathrm{E}$ & G & S & & 2.3 & 0.4 \\
\hline SRC2-3 & $E$ & $\mathrm{~N}$ & $A$ & $\mathrm{~L}$ & $\mathrm{~L}$ & $\mathrm{R}$ & $Y$ & $\mathrm{~L}$ & $\mathrm{~L}$ & $\mathrm{D}$ & $\mathrm{K}$ & $D$ & D & & 1.5 & 0.4 \\
\hline 10 & $\mathrm{R}$ & $\mathrm{H}$ & $\mathrm{K}$ & I & $\mathrm{Lu}^{\mathrm{u}}$ & - & $\mathbf{K}^{\mathrm{u}}$ & $L^{u}$ & $\mathbf{L}$ & $Q$ & $E$ & G & $S$ & & 360 & 47 \\
\hline 11 & $\mathrm{R}$ & $\mathrm{H}$ & $\mathrm{K}$ & 1 & $\mathrm{~L}$ & - & $\mathrm{K}^{\mathrm{u}}$ & Lua & $\mathrm{Lu}^{\mathrm{u}}$ & $Q$ & $E$ & G & $S$ & & 215 & 70 \\
\hline 12 & $\mathrm{R}$ & $\mathrm{H}$ & $\mathrm{K}$ & I & $\mathrm{L}$ & - & $\mathbf{R}^{\mathbf{u}}$ & Lua & $\mathrm{Lu}^{\mathrm{u}}$ & Q & $\mathrm{E}$ & G & $S$ & & 64 & 13 \\
\hline 13 & $\mathrm{E}$ & $\mathrm{N}$ & A & $A^{\text {ua }}$ & $\mathrm{Lu}^{\mathrm{u}}$ & - & $Y^{u}$ & $\mathbf{V u}^{\mathbf{u}}$ & $\mathrm{Nle}^{\mathrm{u}}$ & - & $\mathrm{K}$ & $\mathrm{D}$ & D & & 1.6 & 0.4 \\
\hline 14 & $E$ & $\mathrm{~N}$ & $A$ & $A^{\text {ua }}$ & $\mathrm{Lu}^{\mathrm{u}}$ & - & $Y_{u}^{u}$ & Vu & $\mathrm{Nle}^{\mathrm{u}}$ & & & & & & 26 & 12 \\
\hline 15 & & & & $A^{\text {ua }}$ & $\mathrm{Lu}^{\mathrm{u}}$ & - & $Y^{u}$ & $\mathbf{V u}$ & $\mathrm{Nle}^{\mathrm{u}}$ & - & $\mathrm{K}$ & D & $D$ & & 4.1 & 0.8 \\
\hline 16 & & & & $\mathrm{Lu}^{\mathrm{u} \alpha}$ & $\mathrm{Lu}^{\mathrm{u}}$ & - & $Y^{u}$ & $\mathbf{V}^{\mathbf{u}}$ & $\mathrm{Nle}^{\mathrm{u}}$ & - & $\mathrm{K}$ & $D$ & $D$ & & 2.8 & 0.8 \\
\hline 17 & & & & Lua & $\mathrm{Lu}^{\mathrm{u}}$ & - & $Y^{u}$ & $\mathbf{V}^{\mathbf{u}}$ & $\mathrm{Nle}^{\mathrm{u}}$ & - & $\mathrm{K}$ & $D$ & $D$ & $\mathrm{TK}\left({ }^{*}\right)$ & 1.3 & 0.3 \\
\hline 18 & & & $A^{u}$ & $A^{\text {ua }}$ & $\mathrm{Lu}^{u}$ & - & $Y^{u}$ & $\mathbf{V u}^{\mathbf{u}}$ & $\mathrm{Nle}^{\mathrm{u}}$ & - & $\mathrm{K}$ & $D$ & $D$ & & 1.4 & 0.7 \\
\hline 19 & & & $\mathbf{A}^{\mathrm{u}}$ & $A^{u \alpha}$ & $\mathrm{Lu}^{\mathrm{u}}$ & - & $\mathbf{R}^{\mathbf{u}}$ & $\mathrm{Lu}^{u}$ & $\mathrm{Nle}^{\mathrm{u}}$ & - & $\mathrm{K}$ & $D$ & $D$ & & 0.12 & 0.04 \\
\hline
\end{tabular}

[a] Oligourea inserts are shown in bold. [b] Dissociation constants (KD) and [c] standard deviation value for the interaction of the peptides and hybrids with hVDR LBD. Unlabelled peptides and hybrids were titrated into a fixed concentration of a fluorescently labelled hVDR LBD in the presence of saturating concentrations of calcitriol. * Indicates FITC labelling. For fluorescently labelled hybrid 17, the unlabelled hVDR LBD was titrated into a fixed concentration of the hybrid. Isotherms averaged over three measurements and fitted according to the law of mass action to yield the apparent KD. 

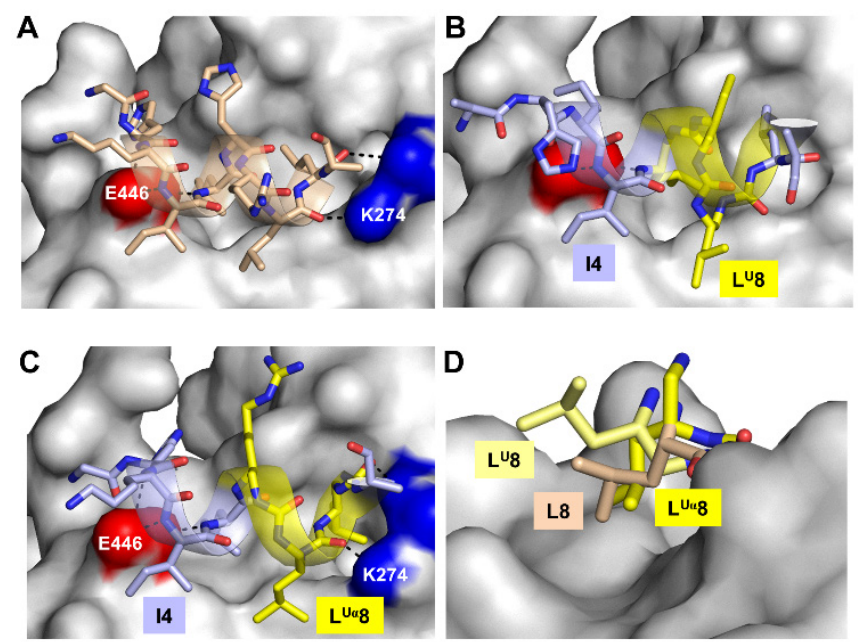

Figure 3. Crystal structures of 10 and 12 in complex with zVDR LBD. A) Structure of bound SRC1-2 (PDB ID: 2HC4) with residues of the charge clamp E446 and K274 shown in red and blue colour, respectively. B) Hybrid 10 bound to zVDR. Hbond interactions with E446 are retained but not those with K274. C) Hybrid 12 bound to VDR and H-bond interactions with E446 and K274. D) Orientations of the iBu side chain at position 8 in SRC1-2 (L8), 10 (Lu8) and 12 (Lua8).

The structures of bound 10-12 (Figures 3 and S19) are helpful to rationalize the decreased binding interaction with these hybrids. Despite the low affinity to zVDR, it occurs that in all three structures, the central part containing the oligourea replacement is helically folded and contacts the protein surface in the expected orientation relative to the two poles of the charge clamp (E446 and K274). We also see that in all three complexes the four hydrophobic side chains superimpose relatively well with those in the natural peptide. However, a closer inspection of the VDR-10 structure reveals that the hybrid is loosely bound at its $\mathrm{C}$-terminus. The iBu side chain of Lu at position 8 is not oriented in an appropriate direction to contact the protein surface (BS\% of Lu8: $1 \%$ compared to $50 \%$ for $L 8$ in SRC1-2) and there is no clear indication that the main chain $\mathrm{C}=\mathrm{O}$ of the same residue establishes a $\mathrm{H}$ bond interaction with K274 (Figure 3B). Interestingly, a tighter interaction with the protein is observed in the structures of 11 and 12 bound to VDR (see Figures $3 C$ and S19). The iBu side chain of the Lua residue which is shifted by one carbon compared to 10 is now pointing towards the protein surface and contributes to the binding as suggested by calculation of its BS\% (50\%, see Figure 3D). Moreover, the $\mathrm{C}=\mathrm{O}$ of Lua in 11 and 12 is $\mathrm{H}$-bonded to $\mathrm{K} 274$ (2.7-2.8 $\AA$ ), mimicking the charge clamp interaction of the native peptide (Figure $3 \mathrm{C}$ ). The structure between VDR and 11 reveals that the lysine side chain Ku7 in 11 provides an additional polar interaction by forming a salt bridge with E285, an interaction which is not present in the structure of SRC1-2 bound to VDR due to the shorter side chain of H6. Notably, a similar salt bridge interaction is formed between R6 and D253 in the structure of SRC2-3 bound to rat VDR.[14] Taken together these results parallel the observations made with the 4-MDM2 structure and underline the importance of tuning the side chain substitution pattern ( $\beta C$ vs $\alpha C$ ) for effective $\alpha$-helix mimicry.

The VDR-13 structure reveals that the short pentaurea helix is topologically equivalent to the central $\alpha$-helix of SRC2-3 in good agreement with the binding assays (Figure 4A). The oligourea helix is fully folded and correctly oriented in the charge clamp with iBu and nBu side chains of Lu5 and Nleu9 residues buried in the hydrophobic groove (BS\%: $75 \%$ and $70 \%$, respectively). The two NHs of the first urea bond are $\mathrm{H}$-bonded $(\mathrm{d}(\mathrm{N}, \mathrm{O})=3.0 \AA$ and $2.8 \AA$ respectively) to $\mathrm{E} 446$ thus mimicking the role of amide NHs of residues $\mathrm{L} 4$ and L5 in SRC2-3. At the negative pole of the helix, the $\mathrm{C}=\mathrm{O}$ of $\mathrm{Vu}$ is engaged in an $\mathrm{H}$-bond interaction with $\mathrm{K} 274$ thus locking the urea helix in the clamp. Interestingly, 
the structure also reveals that the flanking ENA tripeptide at the N-terminus adopts a new conformation - different from that in bound SRC2-3 - that participates in a complex water-mediated network of $\mathrm{H}$-bonds with the oligourea backbone ( $\mathrm{NHs}$ of Aua4 and Yu7) and the protein surface (Figure 4A).
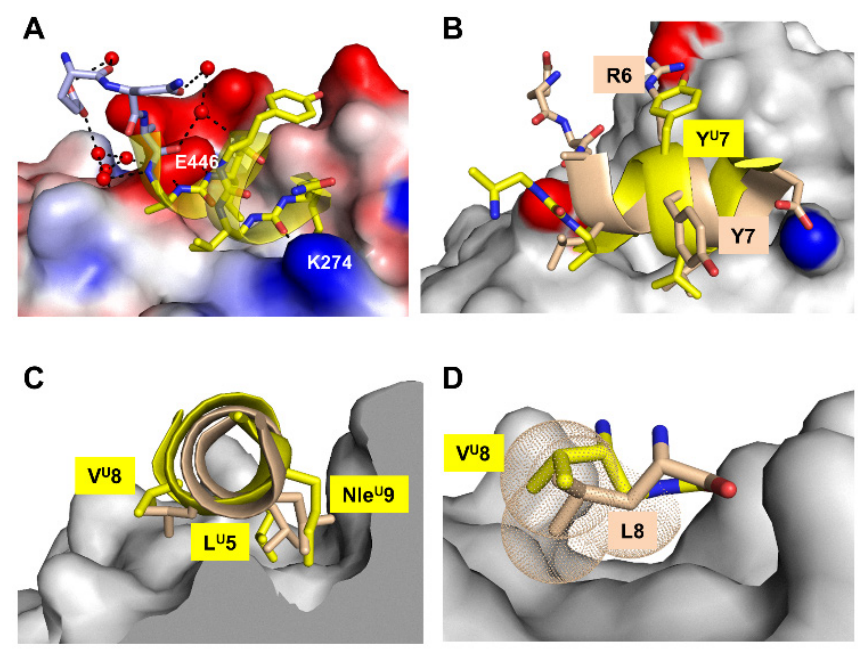

Figure 4. Crystal structures of 13 and 18 in complex with zVDR LBD. A) Ribbon drawing of bound hybrid 13 and details of the water-mediated network of H-bonds. B) Overlay of the structures of SRC2-3 and hybrid 18 (oligourea segment in yellow and SRC2-3 in salmon) highlighting the close orientation of R6 in SRC2-3 and Yu7 in 18. C) Overlay along the helix axis of bound SRC2-3 and hybrid 18 showing key hydrophobic side chains. D) Overlay of L8 and Vu8 side chains in bound SRC2-3 and bound 18 highlighting the increased distance of Vu8 side chain from the surface.

The VDR-18 structure (Figure 4B, 4C and Figure S20) reveals a mode of binding very similar to that of 13 where the side chain of Yu7 actually overlaps reasonably well with R6 in the structure of SRC2-3. Thus, substituting Ru for Yu at position 7 in 18 could potentially add a salt bridge interaction with D253 and increase binding affinity. Furthermore, examination of the structure of 18 bound to VDR reveals little contact between the iPr group of Vu8 and VDR (BS\% $=15 \%$ compared to $57 \%$ for $L 8$ in SRC2-3, Figure 4D) suggesting that extension of the side chain at this position could be beneficial. We thus prepared 19 that combines Ru and Lu residues at positions 7 and 8, respectively. The 10 -fold increase in binding affinity to VDR measured for $19(K D=0.14 \mu \mathrm{M})$ confirmed these elements of design and our ability to generate high affinity ligands of VDR by carefully optimizing the oligourea sequence.

\section{Conclusion}

We report a unique ensemble of X-ray structures of foldamer ligands mimicking $\mathrm{p} 53$ and SRC peptides bound to their respective target proteins MDM2 and VDR. The foldamers under scrutiny are $\mathrm{N}, \mathrm{N}^{\prime}$-linked oligoureas, a class of foldamers which adopt stable helical secondary structures and have been proposed as possible $\alpha$-helix mimics. $[8,25]$ Interfacing oligoureas with peptides was used for the first time in this work to design peptide-oligourea hybrids that disrupt PPIs. High affinity binders were obtained within few rounds of optimization by carefully designing oligourea sequences.

The high resolution structures collected here confirm that a high degree of $\alpha$-helix mimicry was achieved in the two series of ligands. The approach is complementary to $\alpha$-helix mimicry using $\alpha /$ ? peptides.[7d] Whereas [?-amino acid replacements are periodic and follow a regular pattern along the sequence, our approach employs a full oligourea to replace a $\alpha$-helical segment. In most cases the oligourea helix was correctly folded and the initial design hypotheses were validated. The structures show that key hydrophobic contacts are maintained and that the urea backbone is able to 
recreate polar contacts with the protein surface, closely mimicking those formed with the cognate peptide. The difficulty to select the most appropriate side chains in ureido units to mimic a given $\alpha$ helical peptide segment comes in part from the larger diameter of the oligourea helix and from the different spacing of the side chains along the oligourea backbone compared to a canonical $\alpha$-helix. Replacing a peptide by an oligourea segment thus requires key side chains to be specifically adjusted and positioned along the oligourea backbone. Similar to ?]- and $y$-amino acid residues, [7h, 26] the increased diversity in terms of position of substituents in ureido units (e.g. either on one or the other backbone methylene or both) provides modularity and additional opportunities to improve $\alpha$-helix mimicry. This strategy was employed successfully when designing hybrid 6 , a p53 peptide mimic consisting of six $\alpha$-amino acids and four ureido units. In this case, introducing a monomer with an alternative substitution pattern at one position (Lu $\alpha$ ) was critical for $\alpha$-helix mimicry. Another critical element of design is the choice of the side chain to be introduced at a given position. A design principle frequently applied when designing $\alpha$-helix mimics based on $\alpha /$ ? - -peptide is to introduce a ? residue that retain the original side chain.[7d] Conversely, this approach is not always valid for oligoureas and shorter side chains are sometimes more appropriate as they can compensate for the increased diameter of the oligourea helix (e.g. Vu to mimic L in 4, Figure $2 \mathrm{~F}$ ).

By interacting with $\mathrm{N}$ - and $\mathrm{C}$-terminal backbone amides of a short $\alpha$-helical segment, the charge clamp at the surface of VDR precisely selects coregulatory sequences of a given length and can be used as a ruler to compare peptide and foldamer helices. In this respect, X-ray structure analysis of 18 actually reveals that the helical pentaurea segment is a very close mimic of the seven-residue long $\alpha$-helical region of peptide SRC2-3 (Figures 4B and S20).

The strategy reported here, whereby an $\alpha$-helical segment is replaced by a foldamer insert, may thus yield peptide analogues with substantial resistance to proteolytic degradation, a feature which is often desirable when developing peptide therapeutics.[3, 8] We expect this approach to be versatile enough to be combined with other known peptide stabilization methods (e.g. [?-amino acid replacement,[7d] macrocyclization,[4] lipidation[27]) to further increase helical content, potency, and resistance to proteases. The general principles that have been discussed here may thus facilitate lead peptide optimization and the design of more efficient and specific foldamer sequences as disruptors of PPIs or as receptor ligands.

\section{Acknowledgements}

This work was funded in part by the ANR (ANR-15-CE07-0010), by the Conseil Regional de NouvelleAquitaine (2017-1R10115) and by SIRIC Brio. A CIFRE support from UREKA Pharma SAS and ANRT to L. M. is gratefully acknowledged. This work has benefited from the facilities and expertise of IECB Biophysical and Structural Chemistry platform (BPCS), CNRS UMS3033, Inserm US001, Univ. Bordeaux. The authors acknowledge the support and the use of resources of the French Infrastructure for Integrated Structural Biology FRISBI ANR-10-INBS-05, the Instruct-ERIC. The authors thank the staff of Proxima 2 at synchrotron SOLEIL and of ID23 and ID30 at ESRF for assistance in using the beamlines. G. G. is grateful to Céline Reverdy and Jean-Christophe Rain (Hybrigenics Services) for helpful discussions. N. R. thanks Claude Ling and Bruno Kieffer (IGBMC) for NMR measurements of ligand concentrations.

Keywords: Peptidomimetics • Foldamers $\bullet$ Protein-protein interactions $\bullet$ Helical structures $\bullet$ Structure-activity relationships

[1] E. Valeur, M. Guéret Stéphanie, H. Adihou, R. Gopalakrishnan, M. Lemurell, H. Waldmann, N. Grossmann Tom, T. Plowright Alleyn, Angew. Chem. Int. Ed. 2017, 56, 10294-10323. 
[2] a) K. Fosgerau, T. Hoffmann, Drug Discov. Today 2015, 20, 122-128; b) S. Mimmi, D. Maisano, I. Quinto, E. Iaccino, Trends Pharmacol. Sci. 2019, 40, 87-91; c) T. Passioura, T. Katoh, Y. Goto, H. Suga, Annu. Rev. Biochem. 2014, 83, 727-752.

[3] M. Erak, K. Bellmann-Sickert, S. Els-Heindl, A. G. Beck-Sickinger, Bioorg. Med. Chem. 2018, 26, 2759-2765.

[4] C. Morrison, Nat. Rev. Drug Discov. 2018, 17, 531.

[5] A. L. Jochim, P. S. Arora, ACS Chem. Biol. 2010, 5, 919-923.

[6] a) K. Estieu-Gionnet, G. Guichard, Exp. Opin. Drug Discov. 2011, 6, 937-963; b) K. J. Skowron, T. E. Speltz, T. W. Moore, Med. Res. Rev. 2019, 39, 749-770.

[7] a) W. S. Horne, T. N. Grossmann, Nat. Chem. 2020, 12, 331-337; b) M. Pasco, C. Dolain, G. Guichard, in Comprehensive Supramolecular Chemistry II (Ed.: J. L. Atwood), Elsevier, Oxford, 2017, pp. 89-125; c) V. Azzarito, K. Long, N. S. Murphy, A. J. Wilson, Nat. Chem. 2013, 5, 161-173; d) L. M. Johnson, S. H. Gellman, in Methods Enzymol., Vol. 523 (Ed.: E. K. Amy), Academic Press, 2013, pp. 407-429; e) H. Yin, G.-i. Lee, K. A. Sedey, J. M. Rodriguez, H.-G. Wang, S. M. Sebti, A. D. Hamilton, J. Am. Chem. Soc. 2005, 127, 5463-5468; f) W. S. Horne, L. M. Johnson, T. J. Ketas, P. J. Klasse, M. Lu, J. P. Moore, S. H. Gellman, Proc. Nat. Acad. Sci. U.S.A. 2009, 106, 14751-14756; g) R. W. Cheloha, A. Maeda, T. Dean, T. J. Gardella, S. H. Gellman, Nat. Biotech. 2014, 32, 653-655; h) S. Liu, R. W. Cheloha, T. Watanabe, T. J. Gardella, S. H. Gellman, Proc. Nat. Acad. Sci. U.S.A. 2018, 115, 1238312388; i) B. B. Lao, K. Drew, D. A. Guarracino, T. F. Brewer, D. W. Heindel, R. Bonneau, P. S. Arora, J. Am. Chem. Soc. 2014, 136, 7877-7888; j) C. M. Grison, J. A. Miles, S. Robin, A. J. Wilson, D. J. Aitken, Angew. Chem. Int. Ed. 2016, 55, 11096-11100; k) P. Sang, M. Zhang, Y. Shi, C. Li, S. Abdulkadir, Q. Li, H. Ji, J. Cai, Proc. Nat. Acad. Sci. U.S.A. 2019, 116, 10757-10762.

[8] J. Fremaux, C. Venin, L. Mauran, R. H. Zimmer, G. Guichard, S. R. Goudreau, Nat. Commun. 2019, 10, 924.

[9] N. Pendem, C. Douat, P. Claudon, M. Laguerre, S. Castano, B. Desbat, D. Cavagnat, E. Ennifar, B. Kauffmann, G. Guichard, J. Am. Chem. Soc. 2013, 135, 4884-4892.

[10] E. Teyssières, J.-P. Corre, S. Antunes, C. Rougeot, C. Dugave, G. Jouvion, P. Claudon, G. Mikaty, C. Douat, P. L. Goossens, G. Guichard, J. Med. Chem. 2016, 59, 8221-8232.

[11] J. Fremaux, L. Mauran, K. Pulka-Ziach, B. Kauffmann, B. Odaert, G. Guichard, Angew. Chem. Int. Ed. 2015, 54, 9816-9820.

[12] M. Pazgier, M. Liu, G. Zou, W. Yuan, C. Li, C. Li, J. Li, J. Monbo, D. Zella, S. G. Tarasov, W. Lu, Proc. Nat. Acad. Sci. U.S.A. 2009, 106, 4665-4670.

[13] F. Ciesielski, N. Rochel, D. Moras, J. Steroid Biochem. Mol. Biol. 2007, 103, 235-242.

[14] D. Egawa, T. Itoh, A. Kato, S. Kataoka, Y. Anami, K. Yamamoto, Bioorg. Med. Chem. 2017, 25, 568-574.

[15] a) P. Chene, Nat. Rev. Cancer 2003, 3, 102-109; b) K. H. Khoo, C. S. Verma, D. P. Lane, Nat. Rev. Drug Discov. 2014, 13, 217-236; c) Y. Li, J. Yang, A. Aguilar, D. McEachern, S. Przybranowski, L. Liu, C.-Y. Yang, M. Wang, X. Han, S. Wang, J. Med. Chem. 2019, 62, 448-466.

[16] a) A. Y. Belorusova, N. Rochel, in Vitam. Horm., Vol. 100 (Ed.: G. Litwack), Academic Press, 2016, pp. 83-116; b) N. Rochel, F. Molnár, Mol. Cell. Endocrinol. 2017, 453, 22-35. 
[17] J. Xu, R.-C. Wu, B. W. O'Malley, Nat. Rev. Cancer 2009, 9, 615-630.

[18] C. Li, M. Pazgier, C. Li, W. Yuan, M. Liu, G. Wei, W.-Y. Lu, W. Lu, J. Mol. Biol. 2010, 398, 200-

213.

[19] C. Douat-Casassus, K. Pulka, P. Claudon, G. Guichard, Org. Lett. 2012, 14, 3130-3133.

[20] S. A. Kane, C. A. Fleener, Y. S. Zhang, L. J. Davis, A. L. Musselman, P. S. Huang, Anal. Biochem. $2000,278,29-38$.

[21] N. P. Pavletich, K. A. Chambers, C. O. Pabo, Genes Dev. 1993, 7, 2556-2564.

[22] F. Ciesielski, Y. Sato, Y. Chebaro, D. Moras, A. Dejaegere, N. Rochel, J. Med. Chem. 2012, 55, $8440-8449$

[23] a) A. Teichert, L. A. Arnold, S. Otieno, Y. Oda, I. Augustinaite, T. R. Geistlinger, R. W. Kriwacki, R. K. Guy, D. D. Bikle, Biochemistry 2009, 48, 1454-1461; b) N. Rochel, F. Ciesielski, J. Godet, E. Moman, M. Roessle, C. Peluso-Iltis, M. Moulin, M. Haertlein, P. Callow, Y. Mély, D. I. Svergun, D. Moras, Nat. Struct. Mol. Biol. 2011, 18, 564-570.

[24] The zVDR LBD exhibits 79\% similarity (69\% identity) in its sequence with the hVDR LBD, while the ligand binding pocket is conserved both in sequence and structure (see also ref [13]). In addition, the residues of the coactivator binding cleft make similar interaction with coactivator motif.

[25] C. M. Lombardo, V. Kumar M. V, C. Douat, F. Rosu, J.-L. Mergny, G. F. Salgado, G. Guichard, J. Am. Chem. Soc. 2019, 141, 2516-2525.

[26] D. Seebach, A. K. Beck, D. J. Bierbaum, Chem. Biodiv. 2004, 1, 1111-1239.

[27] R. Menacho-Melgar, J. S. Decker, J. N. Hennigan, M. D. Lynch, J.Control. Release 2019, 295, 112. 
